\title{
Evaluation of portable Raman instruments with 532 and 785-nm excitation for identification of zeolites and beryllium containing silicates
}

\author{
Jan Jehlička ${ }^{a *}$ and Peter Vandenabeele ${ }^{b}$
}

\begin{abstract}
In this short paper we present Raman spectra of minerals of the group of zeolites and beryllium containing silicates (thomsonite, stilbite, natrolite, euclase, phenakite, bavenite, milarite and beryl) obtained indoors/outdoors on a collection of samples using a portable 532-nm/785-nm systems. This study complements a previous one in which one of the first handheld 785-nm excitation instruments was tested. To our knowledge this is the first time that a handheld Raman spectrometer equipped with a 532-nm laser as well as a portable dual instrument with $532 / 785-\mathrm{nm}$ excitation was used in mineralogy. For the major findings of the zeolites investigated, the most intense Raman bands are found at correct positions $+/-2-4 \mathrm{~cm}^{-1}$. Although acceptable results were obtained using the 532-nm excitations with both the instruments (the strongest bands are well developed and these bands permit the unambiguous identification), the use of the near-infrared excitation can especially be recommended (higher number of bands at correct positions, spectra with a S/N similar to laboratory spectrometers). Copyright @ 2015 John Wiley \& Sons, Ltd.
\end{abstract}

Keywords: handheld Raman spectrometers; zeolites; beryllium-containing silicates

\section{Introduction}

Lightweight portable or handheld Raman spectrometers have become instruments that can be recommended for common geological fieldwork. As the miniaturization of these instruments has progressed, there are currently lightweight as well as palm-size Raman spectrometers that can nowadays be used for detection of unknown samples including minerals under outdoor conditions. For field geoscience measurements and applications as well as for areas as astrobiology Raman spectroscopy can be proposed as a technique of field detection and identification of minerals ${ }^{[1-5]}$ and biomarkers. ${ }^{[6-11]}$

The approach of recording spectra of minerals for their mostly instantaneous unambiguous identification has been developed using especially the near-infrared diode excitation $(785 \mathrm{~nm}) .{ }^{[3]}$ Critical issues of outdoors work were highlighted recently including diverse analytical issues (collection of scattered light, time of accumulation). ${ }^{[3,4,12,13]}$ Many black and dark minerals are not easy to identify using the portable systems because of absorption of the radiation and/or fluorescence emission. Positioning and focussing of the laser beam onto small grains can be a limiting factor in some cases. ${ }^{[4,13]}$ However some of these observations are, at least at some level, common also when using lab based Raman microspectrometers and related with the properties of the compound. The potential use of the different miniaturized Raman systems available and their use in art, geoscience and forensic areas have been reviewed by Vandenabeele et al. ${ }^{[12]}$ More recently, smaller and robust instruments have been developed, equipped also by less common 1064-nm or 532-nm excitation lasers. The 1064-nm system was evaluated for geoscience and forensic applications, confirming good performance. ${ }^{[7]}$

Zeolites are representatives of tektosilicates-they consist of periodic symmetric arrangements of corner-linked $\mathrm{AlO}_{4}$ and $\mathrm{SiO}_{4}$ tetrahedra combined generally with monovalent or divalent cations. Some of these phases can also by synthetized, and many interesting application features derive from their unusual structure. Silicates and zeolites being common minerals of volcanic and other rocks were successfully investigated in detail since the seventies, and assignments of the Raman bands of common minerals have been carried-out. ${ }^{[14-20]}$ The majority of previous papers on zeolites report data obtained using dispersive laboratory systems with $\mathrm{Ar}^{+}$lasers $(514,532 \mathrm{~nm})$, and also a $488-n m$ excitation ${ }^{[15,18]}$ or $633-n m$ source. ${ }^{[14]}$ Our previous report showed that zeolites could be identified using portable Raman instrumentation equipped with a 785-nm diode. ${ }^{[19]}$ There is a comparison between the performance of laboratory dispersive spectrometers, and near-infrared-excitation portable instrument is reported.

The main goal of the present work is to compare the new types of portable Raman spectrometers for zeolites. First, a new 532-nm laser handheld Raman spectrometer is evaluated; second, performance of a dual instrument-permitting measurements using alternatively both the excitations 785 and $532 \mathrm{~nm}$-is tested. Critical technical aspects are highlighted to permit recommendations for future use of this type of instrumentation for zeolite detection.

\section{Experimental}

\section{Samples}

The same series of zeolites and Be-containing minerals specimens as those investigated previously in our group ${ }^{[3]}$ was studied here. All of

\footnotetext{
* Correspondence to: Jan Jehlička, Charles University, Institute of Geochemistry, Mineralogy and Mineral Resources, Prague, Czech Republic. E-mail: jehlicka@natur.cuni.cz

a Institute of Geochemistry, Mineralogy and Mineral Resources, Charles University, Prague, Czech Republic

b Department of Analytical Chemistry, Raman Research Group, Proeftuinstraat 86, 9000, Ghent, Belgium
} 
the mineral samples investigated in this study were kindly provided from the mineralogical collections of the Institute of Geochemistry, Mineralogy and Mineral Resources at the Faculty of Science, Charles University, Prague, Czech Republic. All the studied specimens are listed in Table 1 (along with their catalogue numbers plus any additional information, such as the locality). Table 1 reports details about the investigated samples. The collection specimens are broadly crystalline, crystal and aggregates dimension around $1-3 \mathrm{~cm}$.

Raman spectra were recorded directly on the mineral specimens, without any previous treatment of the surface. Spectra were recorded indoors, few specimens outdoors as well under cloudy weather. No major difference was found between both the modes of collecting spectra.

\section{Instrumentation}

Three different portable Raman spectrometers were used in this work. Figure 1 presents the instruments and Table 2 their technical

Table 1. List of investigated minerals. All the specimens investigated originate from the mineralogical museum of the Faculty of Science (Charles University in Prague). Archive no. reported

\begin{tabular}{lllr|} 
Mineral & \multicolumn{1}{c}{ Formula } & \multicolumn{1}{c}{ Origin } & Collection \\
\hline Thompsonite & $\mathrm{NaCa}_{2}\left[\mathrm{Al}_{5} \mathrm{Si}_{5} \mathrm{O}_{20}\right] \cdot 6 \mathrm{H}_{2} \mathrm{O}$ & Dobrna, Děčín $\mathrm{CZ}$ & 45321 \\
Stilbite & $\mathrm{Ca} \mathrm{NaCa}_{4}\left[\mathrm{Al}_{8} \mathrm{Si}_{28} \mathrm{O}_{72}\right] \cdot$ & Berufjordur, ICE & 21360 \\
& $\mathrm{n}\left(\mathrm{H}_{2} \mathrm{O}\right)$ & & \\
Natrolite & $\mathrm{Na}_{2}\left[\mathrm{Al}_{2} \mathrm{Si}_{3} \mathrm{O}_{10}\right] \cdot 2 \mathrm{H}_{2} \mathrm{O}$ & Lovozerska, $\mathrm{RU}$ & 17985 \\
Euclase & $\mathrm{BeAl}\left[\mathrm{OH} \mid \mathrm{SiO}_{4}\right]$ & Sardagon, $\mathrm{RU}$ & 18024 \\
Phenakite & $\mathrm{Be}_{2} \mathrm{SiO}_{4}$ & San Miguel, BRA & 5000 \\
Bavenite & $\mathrm{Ca}_{4} \mathrm{Be}_{2} \mathrm{Al}_{2} \mathrm{Si}_{9} \mathrm{O}_{26}$ & Akudertia. Siberia & 18156 \\
& $\left(\mathrm{OH}_{2}\right)_{2}$ & RU & \\
Milarite & $\mathrm{K}_{2} \mathrm{Ca}_{4} \mathrm{Al}_{2} \mathrm{Be}_{4 \mathrm{Si}_{24}}$ & Giuv Tavetsch, $\mathrm{CH}$ & 3295 \\
& $\mathrm{O}_{60} \cdot\left(\mathrm{H}_{2} \mathrm{O}\right)$ & & \\
Beryl & $\mathrm{Be}_{3} \mathrm{Al}_{2} \mathrm{Si}_{6} \mathrm{O}_{18}$ & Prata, IT & Private \\
& & & collection \\
\hline
\end{tabular}

parameters. The first-one, Inspector Raman (Delta Nu), is a 785-nm system different from the first Defender (Ahura) which was applied in the previous investigation of zeolites and berylliumcontaining minerals. ${ }^{[19]}$ The second one is the lightweight 532-nm spectrometer (Rigaku), and finally the third one is the dual laser Raman spectrometer EZRAMAN-I-DUAL Raman system (Enwave Optronics).

The handheld Inspector Raman instrument (mass $1.9 \mathrm{~kg}$ ) was manufactured by Delta Nu at Laramie, USA and equipped with a 785-nm diode laser for excitation with a maximum output power of $120 \mathrm{~mW}$ at source and a thermoelectrically cooled CCD detector with a wavenumber range of $200-2000 \mathrm{~cm}^{-1}$. The instrument is also designed as a handheld pistol-like shape and is operated through the laptop connected via USB cable. The laptop computer is equipped with NuSpec software, which allows the control of experimental settings as well as saving the spectra. Laser power levels at source can be set to five settings: low, medium-low, medium, medium-high and high.

The FirstGuard (Rigaku) instrument weights $2.7 \mathrm{~kg}$ and is equipped with a 532-nm diode laser thermoelectrically cooled CCD detector. The maximum laser power output at source is $60 \mathrm{~mW}$. It operates in the spectral range of $200-3000 \mathrm{~cm}^{-1}$ with the spectral resolution of $10-15 \mathrm{~cm}^{-1}$ as reported by manufacturer. It uses a resistive touch display and allows wider range of experimental settings. In this study the spectra were collected at the following settings: 10 accumulations for $1 \mathrm{~s}$ each and 1 accumulation $10 \mathrm{~s}$ long at the 50-mW laser power.

The portable dual laser Raman spectrometer allowing 532/785$\mathrm{nm}$ excitation is described by Lauwers et al. ${ }^{[20]}$ where it was tested for applications in the field of cultural heritage. This is an EZRAMANI-DUAL Raman system (Enwave Optronics, Irvine CA, USA). The fibre-optic-based spectrometer is equipped with a redion diode laser (785 nm) and a green Nd:YAG laser (532) with interchangeable lenses and adjustable power controller for each laser (maximum output power $400 \mathrm{~mW}$ and $100 \mathrm{~mW}$ for the $785-\mathrm{nm}$ and the 532-nm lasers, respectively). A different grating is used in the spectrometer giving a different spectral range and spectral
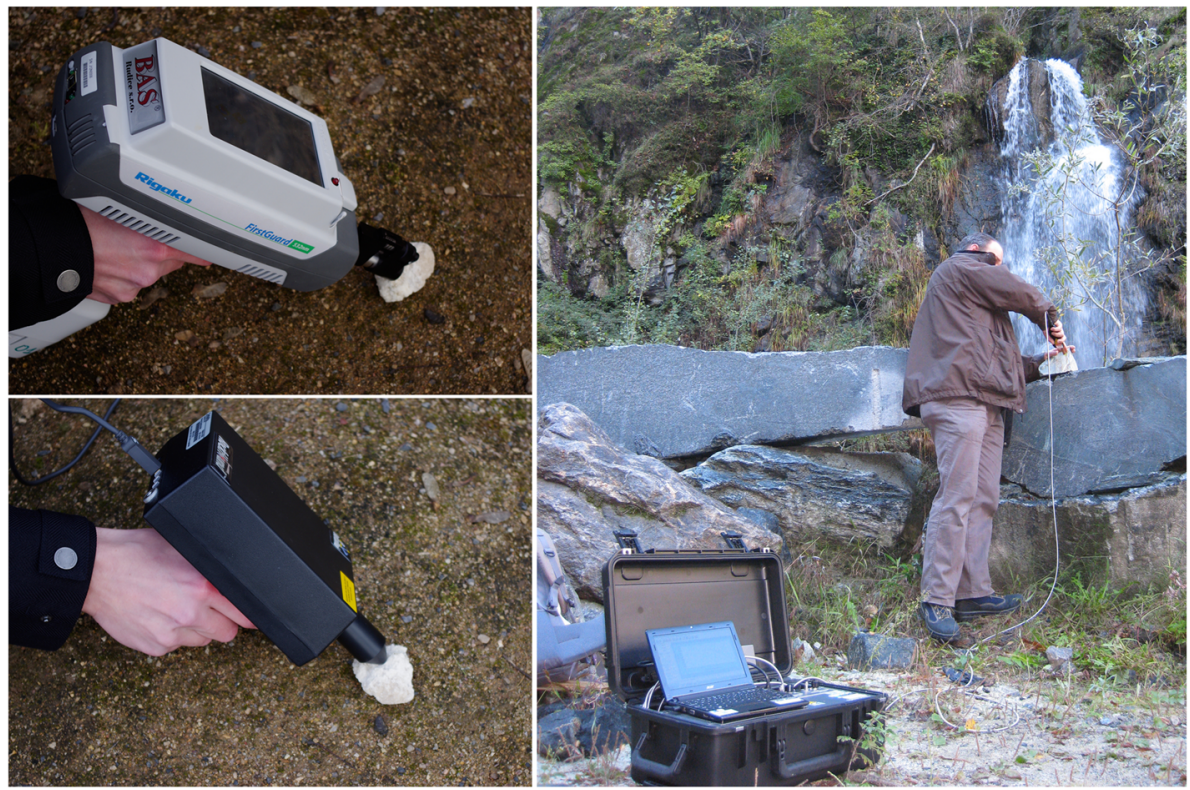

Figure 1. Handheld instruments used in this study. Upper picture 532-nm excitation diode-equipped Raman spectrometer, lower picture 785-nm excitation diode-equipped Raman spectrometer and on the right dual system (532/785-nm excitation). 


\begin{tabular}{|c|c|c|c|c|c|c|c|c|}
\hline Type & $\begin{array}{l}\text { Laser type }(\mathrm{nm}) \\
\text { power }(\mathrm{mW})\end{array}$ & Positioning & Control & $\begin{array}{l}\text { Weight } \\
(\mathrm{kg})\end{array}$ & Head & $\begin{array}{l}\text { Power } \\
\text { source }\end{array}$ & $\begin{array}{c}\text { Spectral } \\
\text { resolution }\left(\mathrm{cm}^{-1}\right)\end{array}$ & Manufacturer \\
\hline \multicolumn{9}{|l|}{ Mobile } \\
\hline EZRaman-I series & $785,532400(50)$ & Manual & Laptop & 12 & Optical fibre & $\begin{array}{l}\text { Rechargeable } \\
\text { Li-ion battery }\end{array}$ & 6 & $\begin{array}{l}\text { Enwave } \\
\text { Optronics Inc }\end{array}$ \\
\hline \multicolumn{9}{|l|}{ Handheld } \\
\hline Inspector Raman & $785,(120)$ & Manual & Laptop & 2.3 & Fixed & $\begin{array}{l}\text { Rechargeable } \\
\text { Li-ion battery }\end{array}$ & 8 & Deltanu \\
\hline First guard & $532,(500)$ & Manual & $\begin{array}{l}\text { Built-in computer } \\
\text { display }\end{array}$ & 2.3 & Fixed & $\begin{array}{l}\text { Rechargeable } \\
\text { Li-ion battery }\end{array}$ & $7-10$ & $\begin{array}{l}\text { San Jose, } \\
\text { USA_Rigaku }\end{array}$ \\
\hline
\end{tabular}

resolution for each laser. The spectrometer can use $230 \mathrm{~V}$ AC or an internal or external Li battery (Enwave Optronics), allowing for higher autonomy.

All instruments provide export of Raman spectra into the Galactic. spc format. Spectra were then worked with using GRAMS Al (Version 8.0, Thermo Electron Corp, Waltham, MA, USA). In some cases Raman spectra are baseline corrected, but no other spectral treatments were undertaken.

Typical data analysis times were 20-60 s, and multiple spectra were obtained for each compound; an optimization of the number of accumulations to improve the signal-to-noise ratio is automatically carried out by the software. The spectrometer automatically

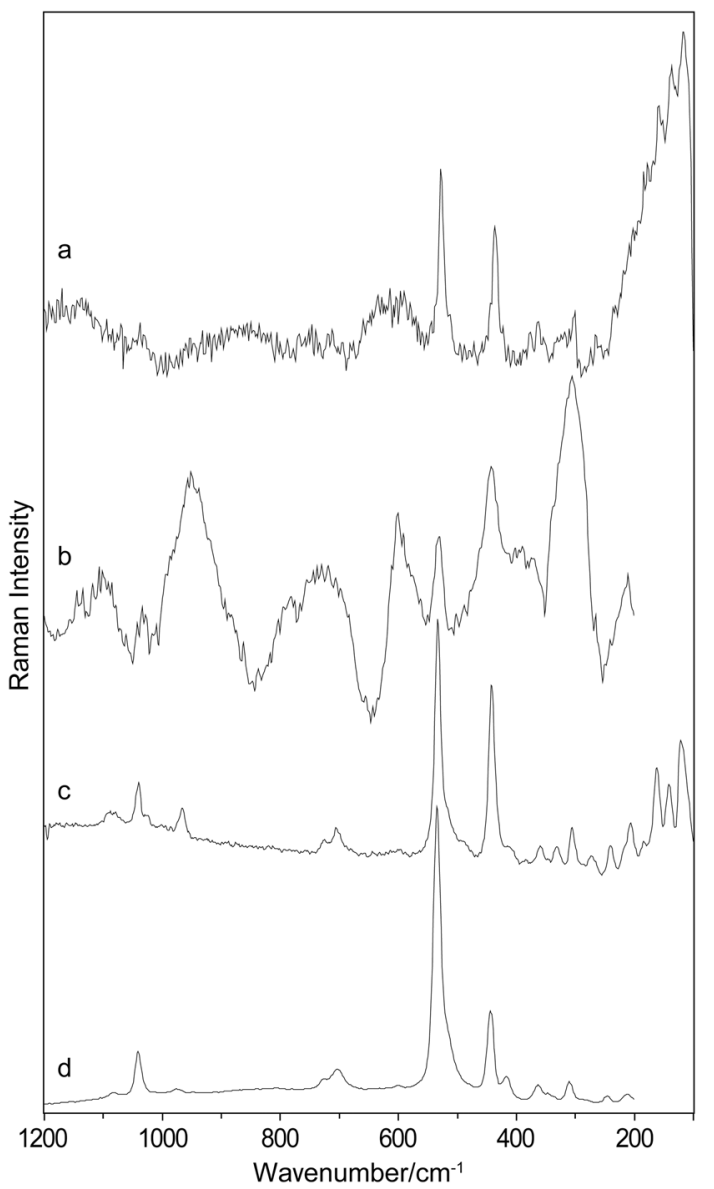

Figure 2. Raman spectra of natrolite obtained using the portable Raman spectrometers-baseline corrected spectra. Envawe $532 \mathrm{~nm}$ (a), Rigaku $532 \mathrm{~nm}$ (b), Envawe $785 \mathrm{~nm}$ (c) and DeltaNu $785 \mathrm{~nm}$ (d). records a dark spectrum and subtracts this from the raw data to correct for signals internally caused by the spectrometer. Based on previous experience all outdoor measurements were carried out by careful shielding of the specimens and instrument measurement area using a black cloth. The identifications of minerals by portable instruments is based on comparisons of data with reference values reported in literature. For any problematic spectra (faint signals, fluorescence complications and bigger difference in wavenumbers obtained) and for better general future applications we collected spectra on the same specimens using a lab Raman microspectrometer. Obtaining at least half of the strong and medium intensity bands at correct positions $+/-3 \mathrm{~cm}^{-1}$ was considered as sufficient for acceptable identification.

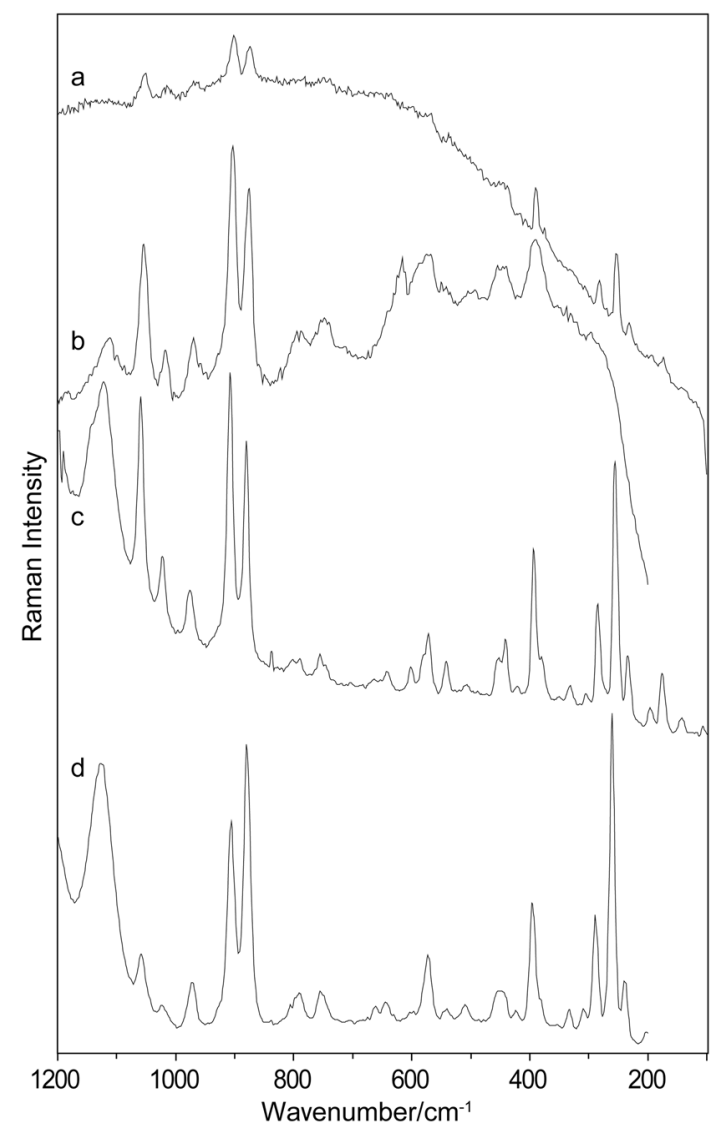

Figure 3. Raman spectra of euclase obtained using the portable Raman spectrometers-uncorrected baseline. Envawe $532 \mathrm{~nm}$ (a), Rigaku $532 \mathrm{~nm}$ (b), Envawe $785 \mathrm{~nm}$ (c) and DeltaNu $785 \mathrm{~nm}$ (d). 


\section{Results and discussion}

It was shown in previous reports that detection of minerals using portable Raman instruments can generally be achieved unambiguously and fast. This work was concerned with the identification of minerals from the following mineral groups: sulfates, carbonates, oxides and silicates. Critical issues of the successful application were identified as follows: absorption and fluorescence effects in the case of dark coloured minerals, measurements of very small grains in the frame of fine-grained rocks, as well as stable positioning. Here, we would like to estimate the performance of a given instrumentation where the 532-nm lasers are used.

Figures 2-4 present spectra obtained using different instruments and wavelength of excitation. Tables 3 and 4 summarize the Raman bands obtained analysing the minerals specimens using the handheld and portable instruments as well as comparisons with reference data from laboratory microspectrometer collected from Jehlička et al. ${ }^{[19]}$ Here, information about the strongest bands found at correct positions $+/-3 \mathrm{~cm}^{-1}$ are reported (number of bands and wavenumbers).

Figures 2-4 show different Raman spectra of natrolite, euclase and phenakite obtained using the portable and handheld Raman spectrometers. Spectra of natrolite are reported as baselinecorrected, spectra of euclase as recorded. In both the figures, the two upper spectra are those obtained using the 532-nm excitation, the lower two those with 785-nm excitation.

Generally speaking the quality of spectra obtained is good or even excellent in the case of both the 785-nm cases: excellent $\mathrm{S} / \mathrm{N}$, acceptable baseline, the wavenumbers obtained differ $+/-2 \mathrm{~cm}^{-1}$ comparing to the reference values. Excellent coherence between

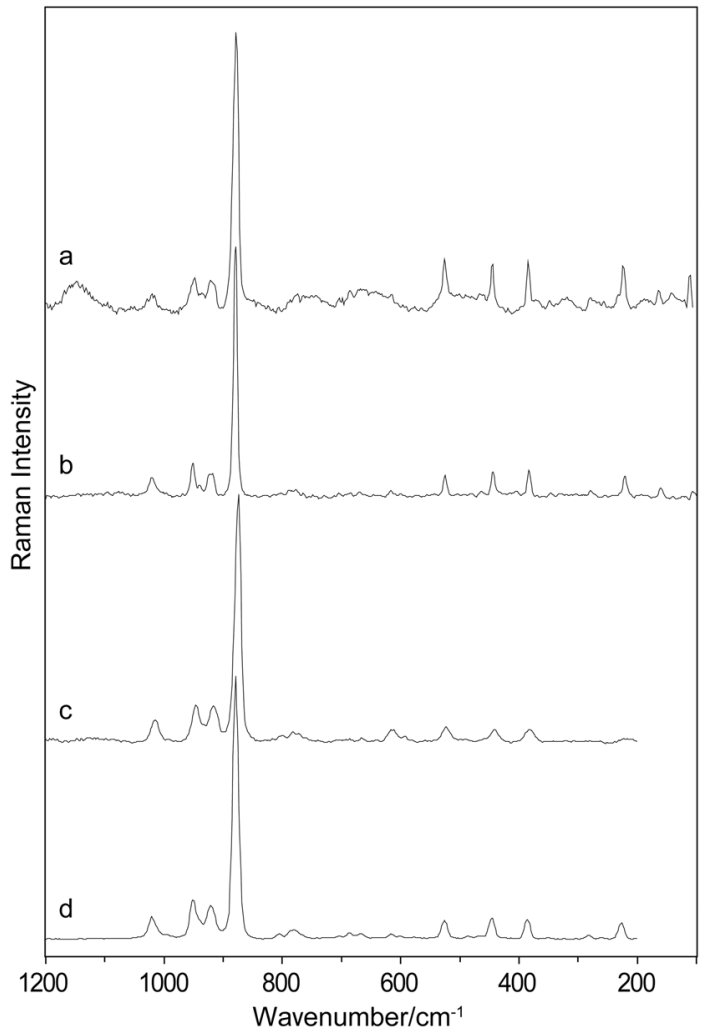

Figure 4. Raman spectra of phenakite obtained using the portable Raman spectrometers - uncorrected baseline. Envawe $532 \mathrm{~nm}$ (a), Envawe $785 \mathrm{~nm}$ (b), Rigaku $532 \mathrm{~nm}$ (c) and DeltaNu 785 nm (d).

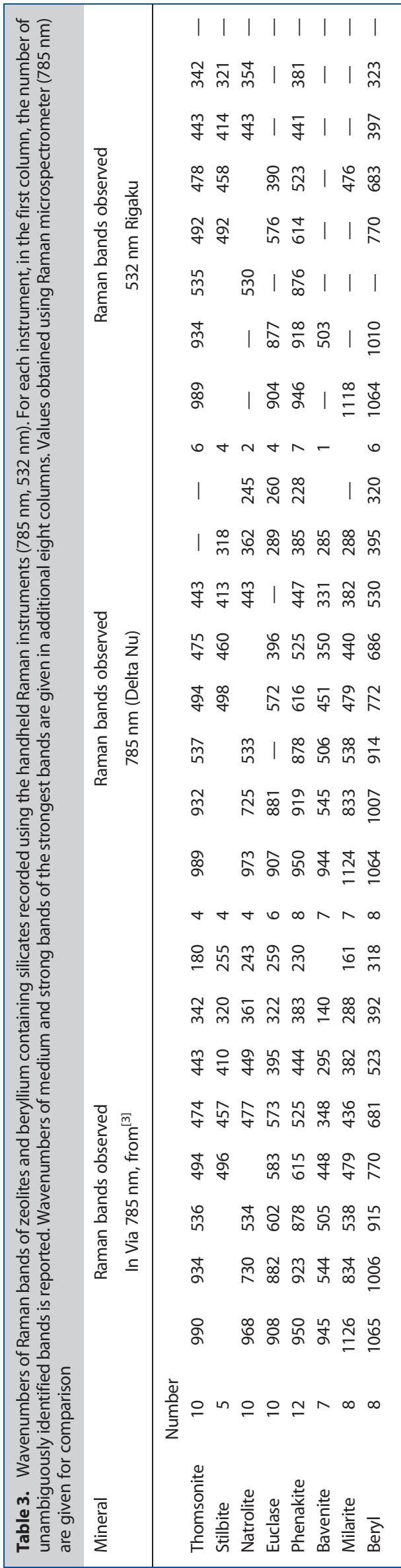




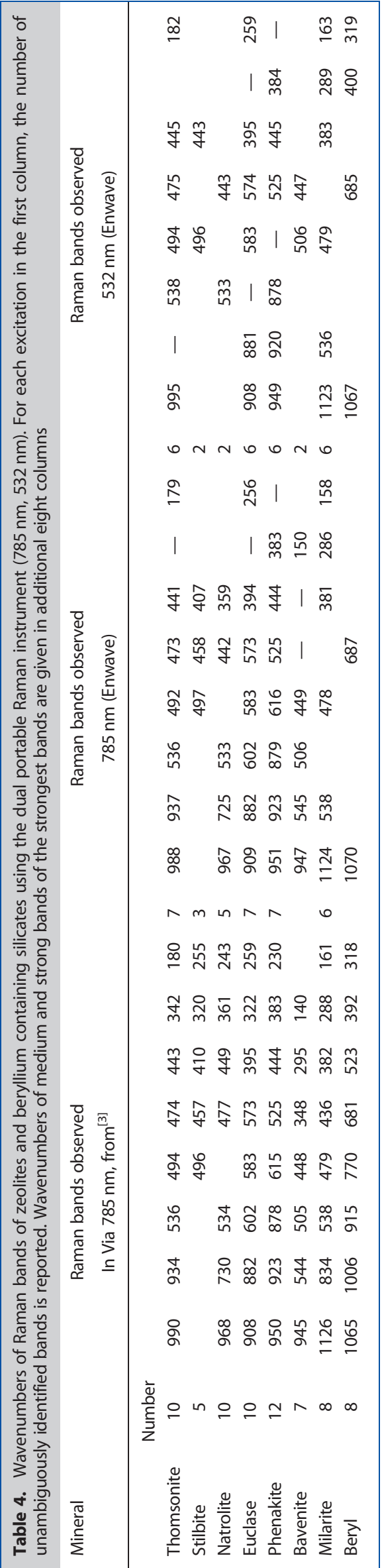

spectra obtained using the laboratory Raman microspectrometer and portable systems can be observed in the case of good Raman scatterers as in the case of euclase and phenakite. The worse situation can be seen for natrolite, even if also in this case the strongest bands are well recorded, however in this case sometimes weak and also non systematically shifted. In this case, an unacceptable difference up to around $9 \mathrm{~cm}^{-1}$ was observed using all the instruments tested. In Fig. $4 a$ and $b$ spectra from the dual spectrometer are reported showing the possibility of recording also the bands below $200 \mathrm{~cm}^{-1}$, which is the advantage comparing to the Delta Nu spectrometer (6 bands at correct positions). Here, coherent detection of these minerals using instruments equipped by $785 \mathrm{~nm}$ confirms the previous report. ${ }^{[19]}$

The 532-nm equipped portable and handheld spectrometers used in this study perform more ambiguous results. In the case of the Rigaku handheld system, additional not-yet solved technical problems are behind artefacts observed. This can be seen in Fig. 1 where the strong wavy artefact feature, which is characteristic and omnipresent, occurs, generally on faint Raman scatterers. On the contrary, when studying other types of compounds, i.e. carotenoids, excellent and correct spectra are obtained. However, in this case, resonance Raman effects are observed allowing very good performance on such compounds. Sometimes, even the 532-nm excitation allows the acquisition of very good spectra and also correct identification. However, the difference between obtained wavenumbers and the reference values can exceed $3 \mathrm{~cm}^{-1}$.

In Fig. 5 baseline uncorrected Raman spectra of milarite obtained with the 785-nm excitation are reported. The upper spectrum corresponding to the Enwave instrument is of good quality with Raman bands at correct positions (eight unambiguous bands). Interestingly it is possible to record also the bands below $200 \mathrm{~cm}^{-1}$, which is the advantage comparing to the Delta Nu spectrometer (six unambiguous bands).

Few general conclusions can be noted comparing spectra reported here in Figs. 2-5 and comparing results in Tables 3 and 4. Both the instruments with near-infrared excitation can provide better

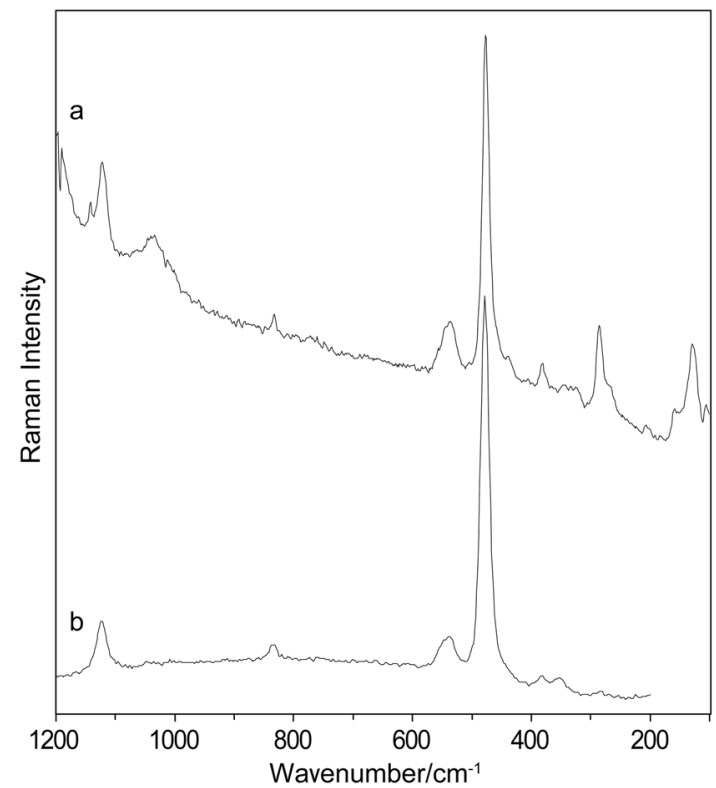

Figure 5. Raman spectra of milarite obtained using the portable Raman spectrometers-uncorrected baseline. Envawe $785 \mathrm{~nm}$ (a) and DeltaNu $785 \mathrm{~nm}$ (b). 
quality spectra and higher number of Raman bands observed at correct $\left(+/-3 \mathrm{~cm}^{-1}\right)$ positions. This seems somehow unexpected as well as the problematic performance of the green laser excitation instrument. Testing of few other instruments is currently carried-out to better address the properties and technical features of those handheld systems.

\section{Conclusions}

Portable Raman spectrometers equipped with near-infrared-excitation diodes seems to be optimal instruments for discriminating zeolites and beryllium-containing silicates. Generally speaking, the main Raman bands of the investigated minerals are found at correct wavenumber positions, compared to the literature reference values and previous studies with lab Raman microspectrometers with excellent spectral resolution $\left(+/-2-6 \mathrm{~cm}^{-1}\right)$. The portable Raman instruments tested in this study are useful tools for mineralogical purposes. This study complements previous investigations where Raman spectroscopy has been identified as a useful tool for identification of many minerals of different groups (sulphates, carbonates, silicates and organic minerals) outdoors and also in the frame of outcrops. Spectrometers allowing excitation with 532-nm lasers can also be used; however, their performance is clearly lower comparing to the 785-nm excitation. In this case some minerals can somehow ambiguously be identified using only few strongest Raman bands (this concerns namely bavenite, natrolite, and stilbite).

\section{Acknowledgements}

This work was supported by grant no. P210/10/0467 from the Grant Agency of the Czech Republic and by institutional support MSM0021620855 from the Ministry of Education of the Czech Republic (to JJ). We thank Adam Culka for technical assistance during manuscript fine-tuning.

\section{References}

[1] J. Jehlička, P. Vítek, H. G. M. Edwards, M. D. Hargreaves, T. Čapoun, J. Raman Spectrosc. 2009, 40, 1082.

[2] J. Jehlička, P. Vítek, H. G. M. Edwards, M. D. Hargreaves, T. Čapoun J. Raman Spectrosc. 2009, 40, 1645.

[3] J. Jehlička, A. Culka, P. Vandenabeele, H. G. M. Edwards, Spectrochim. Acta, Part A 2011, 80, 36.

[4] P. Vitek, J. Jehlička,H. G. M. Edwards, Appl. Spectrosc. 2013, 67, 767.

[5] A. Culka,F. Košek, P. Drahota, J. Jehlička, Icarus 2014, 243, 440.

[6] J. Jehlička, H. G. M. Edwards, A. Culka, Phil. Trans. Roy. Soc. A 2010, 368 , 3109.

[7] P. Vítek, E. M. A. Ali, H. G. M. Edwards, J. Jehlička, R. Cox, K. Page, Spectrochim. Acta, Part A 2012, 86, 320.

[8] A. Culka, J. Jehlička, P. Vandenabeele, H. G. M. Edwards, Spectrochim. Acta, Part A 2011, 80, 8.

[9] P. Vítek, J. Jehlička, H. G. M. Edwards, I. Hutchinson, C. Ascaso, J. Wierzchos, Astrobiology 2012, 12, 1095.

[10] J. Jehlička, A. Oren, J. Raman Spectrosc. 2013, 43, 1275.

[11] I. Miralles, S. E. Jorge-Villar, Y. Canton, F. Domingo, Astrobiology 2012 $12,743$.

[12] P. Vandenabeele, J. Jehlička, P. Vitek, H. G. M. Edwards Planet. Space Sci. 2012, 62, 48

[13] P. Vandenabeele, H. G. M. Edwards, J. Jehlička, Chem. Rev. Roy. Soc. 2014, 43, 2628.

[14] W. P. Griffith, J. Chem. Soc. A, Inorg. Phys.Theor. 1969, 9, 1372.

[15] B. Wopenka, J. J. Freeman, T. Nikischer, Appl. Spectrosc. 1998, 52, 54.

[16] G. D. Gatta, V. Kahlenberg, R. Kaindl, N. Rotiroti, P. Cappelletti, M. de Gennaro, Am. Mineral. 2010, 95, 495.

[17] R. L. Frost, A. Lopez, L. Wang, A. W. Romano, R. Scholz, Spectrochimica Acta Part A 2015, 137, 70.

[18] I. Moroz, M. Roth, M. Boudeulle, G. Panczer, J. Raman Spectrosc. 2000, $31,485$.

[19] J. Jehlička, P. Vandenabeele, H. G. M. Edwards, Spectrochimica Acta Part $A, \mathbf{2 0 1 2}, 86,341$.

[20] D. Lauwers, A. G. Hutado, V. Tanevska, L. Moens, D. Bersani, P. Vandenabeele, Spectrochim. Acta. A. 2014,118, 294. 